\title{
BMJ Open Study protocol for a randomised controlled trial: treatment of early intrauterine growth restriction with low molecular weight heparin (TRACIP)
}

Edurne Mazarico, ${ }^{1,2,3}$ Anna Peguero, ${ }^{4}$ Marta Camprubí, ${ }^{1}$ Carlota Rovira,
Maria Dolores Gomez Roig, ${ }^{1,2,3}$ Daniel Oros, ${ }^{3,6}$ Patricia Ibáñez-Burillo, ${ }^{6}$
Jon Schoorlemmer, ${ }^{7}$ Narcís Masoller, ${ }^{4}$ Maria Dolors Tàssies, ${ }^{8}$ Francesc Figueras ${ }^{4}$

To cite: Mazarico E, Peguero A, Camprubí M, et al. Study protocol for a randomised controlled trial: treatment of early intrauterine growth restriction with low molecular weight heparin (TRACIP). BMJ Open 2018;8:e020501. doi:10.1136/ bmjopen-2017-020501

- Prepublication history for this paper is available online. To view these files, please visit the journal online (http://dx.doi org/10.1136/bmjopen-2017020501).

Received 7 November 2017

Revised 7 May 2018

Accepted 12 June 2018

Check for updates

(C) Author(s) (or their employer(s)) 2018. Re-use permitted under CC BY-NC. No commercial re-use. See rights and permissions. Published by BMJ.

For numbered affiliations see end of article.

Correspondence to Dr Francesc Figueras; FFIGUERA@clinic.cat

\section{ABSTRACT}

Introduction The incidence of intrauterine growth restriction (IUGR) is estimated at about $3 \%$ of pregnancies, and it is associated with $30 \%$ of all perinatal mortality and severe morbidity with adverse neurodevelopmental and cardiovascular health consequences in adult life. Early onset IUGR represents $20 \%-30 \%$ of all cases and is highly associated with severe placental insufficiency. The existing evidence suggests that low molecular weight heparin (LMWH) has effects beyond its antithrombotic action, improving placental microvessel structure and function of pregnant women with vascular obstetric complications by normalising proangiogenic and antiapoptotic protein levels, cytokines and inflammatory factors. The objective of our study is to demonstrate the effectiveness of LMWH in prolonging gestation in pregnancies with earlyonset IUGR.

Methods and analysis This is a multicentre, tripleblind, parallel-arm randomised clinical trial. Singleton pregnancies qualifying for early (20-32 weeks at diagnosis) placental IUGR (according to Delphi criteria) will be randomised to subcutaneous treatment with bemiparin $3500 \mathrm{IU} / 0.2 \mathrm{~mL} /$ day or placebo from inclusion at diagnosis to the time of delivery. Analyses will be based on originally assigned groups (intentionto-treat). The primary objective will be analysed by comparing gestational age and prolongation of pregnancy (days) in each group with Student's t-tests for independent samples and by comparing KaplanMaier survival curves (from inclusion to delivery, logrank test). A linear regression model for gestational age at birth will consider the following covariates: gestational age at inclusion (continuous) and preeclampsia (binary).

Ethics and dissemination The study will be conducted in accordance with the principles of Good Clinical Practice. This study was approved by the Clinical Research Ethics Committee (CEIC) of Sant Joan de Déu Hospital, on 13 July 2017. The trial is registered in the public registry www.clinicaltrial.gov. according to Science Law 14/2011, and the results will be published in an open access journal.

Trial registration number NCT03324139; Pre-results.
Strengths and limitations of this study

- This is a multicentre, triple-blinded, randomised trial with two parallel groups.

- The primary outcome (prolongation of pregnancy) is a surrogate of perinatal health

- The sample size limits the analysis of secondary outcomes.

Follow-up of the offspring is limited to the neonatal period.

\section{INTRODUCTION}

\section{Background}

Placental insufficiency and intrauterine growth restriction

Intrauterine growth restriction (IUGR) is defined as a failure to achieve the endorsed growth potential secondary to placental insufficiency. Its incidence is estimated at about $3 \%$ of pregnancies, and it is associated with $30 \%$ of all perinatal mortality and severe morbidity, ${ }^{1}$ with adverse neurodevelopmental and cardiovascular health consequences in adult life.

Early onset IUGR represents $20 \%-30 \%$ of all $\mathrm{IUGR}^{1}$ and presents in association with early pre-eclampsia (PE) in up to $50 \%$ of cases. ${ }^{1}$ This type of IUGR is highly associated with severe placental insufficiency; subsequent chronic fetal hypoxia is the major contributor to poor perinatal outcomes. ${ }^{2}$

The key to successful placental implantation is controlled activation of the haemostatic system which balances the process of trophoblastic invasion and protects against bleeding. ${ }^{3}$ In PE and early onset IUGR, this vascular remodelling does not exist or is incomplete ${ }^{2}$ which leads to placental hypoperfusion associated with endothelial cell dysfunction and subsequent coagulation, fibrin deposition and uteroplacental 
thrombosis with local implications in the case of IUGR and systemic implications in the case of PE. ${ }^{4}$ Pathological examination of the placenta shows uteroplacental thrombosis, infarctions and fibrin deposits.

The natural history of placental insufficiency decreases fetal well-being, with a progression to hypoxia and acidosis; in clinical practice, this deterioration is monitored by Doppler study of the umbilical artery (which reflects the placental lesion) and ductus venosus (which reflects fetal cardiac diastolic failure secondary to acidosis) to individually time the delivery in an elective and adapted manner. The latency time to severe fetal deterioration is variable but is usually $3-4$ weeks. ${ }^{5}$ It is estimated that between 24 and 28 weeks, each week of prolonged gestation increases survival without sequelae by $10 \%-15 \%$; and between 28 and 32 weeks, it increases by $5 \%{ }^{6}$ The objective of fetal monitoring is therefore to maximise gestational age at birth while minimising the risk of death and sequelae.

\section{Heparin and placenta: biological plausibility}

There is in-vitro evidence that heparin improves placental perfusion and stimulates neoangiogenesis. Heparin exerts its anticoagulant function through the union and potentiation of antithrombin, and it also releases tissue inhibitory factor to the circulation. ${ }^{2}$ It also has beneficial effects through other routes that do not have to do with its anticoagulant effect ${ }^{7}$ : it influences trophoblast growth and development, it decreases apoptosis, it acts as an indirect growth factor and it diminishes inflammation via its anticomplement effects. ${ }^{89}$

Significant increases in the levels of some proteins that play important roles in placental development and angiogenesis were reported in patients treated with low molecular weight heparin (LMWH), such as leptin, Ang-2, vasular endotelial growth factor receptor (VEGFR)-3, angiostatin, tissues inhibitors (TIMP-1) and tumoral necrosis factor $(\mathrm{TNF}-\alpha)^{10-12}$

In-vitro results indicate that heparin normalises matrix metalloproteinases (MMP-9) levels at the level of placental microvessels. ${ }^{8}$ MMP-9 controls trophoblastic invasion, and the decrease of its expression in trophoblasts as a response to hypoxia reduces invasiveness which can impair placenta implantation. LMWH significantly increases the levels of both pro-MMP and active forms which increases trophoblast invasiveness and promotes angiogenesis in vitro.

Heparin also inhibits placental apoptosis by increasing cellular protection ${ }^{13}$ and normalises levels of inflammatory factors (interferon (IFN- $\gamma$ ), transforming growth factor (TGF- $\beta 1$ ), interleukin (IL) 6, IL-8) that are elevated in the microvessels of pregnant women with vascular complications.

Much of the available evidence comes from in-vitro models; the clinical correlations of these effects described in experimental models are poorly characterised.

These data suggest that LMWH has effects beyond its antithrombotic action, improving the microvessel structure and function of pregnant women with vascular obstetric complications by normalising levels of proangiogenic and antiapoptotic proteins, cytokines and inflammatory factors at the microvessel level. These changes facilitate appropriate invasion and angiogenesis activity to promote endothelial cell and trophoblast survival.

\section{Heparin and placenta: clinical evidence}

There are few high-quality clinical studies on the efficacy of heparin in gestation. A review ${ }^{14}$ involving 10 randomised trials with 1139 patients concluded that for women at high risk of complications secondary to placental insufficiency, treatment with heparin was associated with significant reductions in perinatal mortality, prematurity before 34 and 37 weeks and low fetal weight for gestational age. However, these studies only include pregnant women at risk, mainly with a history of placental insufficiency in a previous pregnancy. Another recent meta-analysis ${ }^{15}$ (including six clinical trials) showed that in high-risk pregnant women treated primarily with aspirin, the addition of LMWH reduced the incidences of $\mathrm{PE}$ and low birth weight for gestational age by $\sim 50 \%$. However, another large individual patient meta-analysis failed to find any benefit of heparin in women with highrisk pregnancy. ${ }^{16}$

Despite the evidence of potential benefit for secondary prevention of placental insufficiency and its complications, there is scant and contradictory evidence about its benefit in pregnant women who already have placental insufficiency. It would be in this clinical indication, which is associated with greater complication risk and need for preterm birth, that the necessary number of patients to treat may prove to be more beneficial. Four clinical trials were performed in women with placental insufficiency during gestation, and they employed heterogeneous diagnostic criteria and methodological aspects and results. Kingdom et $a l^{17}$ and Souza $e t a l^{18}$ randomised patients with clinical criteria for placental insufficiency who were treated with heparin or placebo and did not observe significant differences in gestational age at birth (35.6 weeks vs 34.3 weeks and 35.69 weeks vs 33.5 weeks, respectively) or perinatal morbidity and mortality. In contrast, Yu et al $^{19} 20$ demonstrated significant differences in gestational age at birth (37 weeks vs 38 weeks and 36.2 weeks vs 37.1 weeks) and fetal birth weight in patients with placental insufficiency treated with heparin.

\section{Study justification}

There is good biological evidence on the potential beneficial effects of heparin on the placenta, but the clinical correlation of the effects described in experimental models is lacking. In addition, the clinical evidence of its benefit to treat gestation already complicated with placental insufficiency is weak and contradictory. Heparin has the potential to prolong gestation during a clinically important period to maximise gestational age at birth to reduce mortality and perinatal sequelae. 


\section{HYPOTHESIS}

\section{Primary hypothesis}

Treatment with LMWH at the time of diagnosis of early onset IUGR prolongs the latency to severe fetal impairment, increasing the gestational age at delivery of these patients.

\section{Secondary hypothesis}

i. Treatment with LMWH at the time of diagnosis of early onset IUGR reduces neonatal morbidity.

ii. Treatment with LMWH at the time of IUGR diagnosis improves maternal and placental biochemical, angiogenic and inflammatory profiles.

iii. Treatment with LMWH at the time of diagnosis of early-onset IUGR reduces placental lesions secondary to poor placental and fetal perfusion.

\section{OBJECTIVES}

\section{Primary objectives}

To demonstrate the effectiveness of LMWH in prolonging gestation in pregnancies with early-onset IUGR.

\section{Secondary objectives}

i. To demonstrate the effectiveness of LMWH in reducing neonatal morbidity in pregnancies with early-onset IUGR.

ii. To demonstrate the effect of LMWH in improving proangiogenic and anti-inflammatory maternal and placental profiles in pregnancies with early-onset IUGR.

iii. To demonstrate the effects of LMWH in reducing thrombotic and ischaemic placental lesions.

\section{TRIAL DESIGN}

This is a multicentre, triple-blinded, randomised, phase III trial with two groups in parallel.

\section{METHODS}

\section{Study setting}

The study will be conducted within Spanish academic hospitals with specialist experience in managing IUGR: Hospital Sant Joan de Déu (Barcelona), Hospital Clínic (Barcelona) and Hospital Clínico Lozano Blesa (Zaragoza). The first two hospitals are legally integrated in the Center of Maternal Fetal and Neonatal Medicine of Barcelona (BCNatal). Each of these hospitals has over 3000 deliveries and performs more than 10000 fetal ultrasounds annually.

\section{ELIGIBILITY CRITERIA}

\section{Inclusion criteria}

\section{Singleton fetus.}

II. Diagnosis of early onset IUGR according to the Delphi classification ${ }^{21}$ (20-32 weeks at diagnosis) with umbilical artery Doppler with absent/reversed dia- stolic flow; or estimated fetal weight $<10$ th percentile plus pulsatility index (PI) of umbilical artery Doppler $>95$ th percentile or estimated fetal weight $<10$ th percentile plus mean PI of uterine artery Doppler $>95$ th percentile.

III. The weeks for inclusion will be between 20-32 weeks of gestation, at the moment of the diagnosis.

\section{Exclusion criteria}

I. Abnormal karyotype, structural abnormalities or congenital infections.

II. Treatment with LMWH or oral anticoagulants prior to inclusion.

III. History of heparin-induced thrombocytopenia.

IV. Active haemorrhage or increased risk of bleeding due to haemostatic changes.

V. Severe hepatic or pancreatic dysfunction.

VI. Organic lesions that can bleed (eg, active peptic ulcer, haemorrhagic stroke, aneurysm or brain tumour).

\section{Stop treatment criteria}

I. Intolerance or hypersensitivity to bemiparin.

II. Allergic reaction.

III. Clinically relevant bleeding (requiring hospitalisation).

IV. Requirement of LMWH per clinical indication.

\section{INTERVENTION}

Patients who agree to participate and provide signed informed consent will be centrally randomised (via a website) through the Clinical Trials Unit (CTU) of Sant Joan de Déu Hospital in Barcelona (block randomisation of 10) to $3500 \mathrm{IU} / 0.2 \mathrm{~mL} /$ day of subcutaneous (sc) bemiparin sc or placebo of the same presentation as the active drug, from inclusion to delivery (estimated median of 5-6 weeks, maximum of 17 weeks).

The treatment will be delivered to the patients weekly using the same presentation and labelled with the study identification and participant number.

\section{CONTROL VARIABLES}

1. Maternal age at birth; continuous (years).

2. Smoking during gestation; continuous (cigarettes/ day).

3. Maternal weight at the beginning of gestation; continuous $(\mathrm{kg})$.

4. Maternal height; continuous $(\mathrm{cm})$.

5. Maternal ethnic origin; categorical (Europe, Africa, South America, Maghreb, Asia, Others).

6. Parity (number of deliveries $>22$ weeks); discrete.

7. History of $\mathrm{PE}^{22}$; binary (yes $/ \mathrm{no}$ ).

8. History of gestational hypertension; binary (yes/no).

9. History of IUGR (birth weight below the 10th percentile) ${ }^{23}$; binary (yes/no).

10. Gestational age at the inclusion in the study; continuous (weeks). 
11. Diastolic blood pressure (DBP) at inclusion; continuous $(\mathrm{mm} \mathrm{Hg}$ ).

12. Systolic blood pressure (SBP) at inclusion; continuous ( $\mathrm{mm} \mathrm{Hg}$ ).

13. Mean PI of the uterine arteries at inclusion and before delivery; continuous (normalised by gestational age) ${ }^{24}$

14. PI of the umbilical artery at inclusion and before delivery; continuous (normalised by gestational age) ${ }^{25}$

15. PI of the middle cerebral artery at inclusion and before delivery; continuous (normalised by gestational age). ${ }^{26}$

16. PI of the ductus venosus at inclusion and before delivery; continuous (normalised by gestational age). ${ }^{27}$

17. Hypertension in current pregnancy; binary (yes/no).

18. PE in current pregnancy; binary (yes/no).

\section{OUTCOMES}

\section{Primary outcomes}

Gestational age at live birth (dated by ultrasound $<14$ weeks by measurement of crown rump length; continuous $(\mathrm{mm}){ }^{28}$

Prolongation of pregnancy: time from inclusion to live birth.

\section{Secondary outcomes}

1. IUGR: birth weight less than the 10th percentile for our population ${ }^{23}$ with PI of the umbilical artery during the third trimester (on two separate occasions $>48$ hours) higher than the 95 th percentile ${ }^{25}$; binary (yes/no).

2. $\mathrm{PE}^{22}$ : $\mathrm{DBP} \geq 90 \mathrm{~mm} \mathrm{Hg}$ and/or $\mathrm{SBP} \geq 140$ in two separate determinations, $>4$ hours with proteinuria $>300 \mathrm{mg} / 24$ hours or other maternal organ dysfunction/renal insufficiency, liver involvement, neurological complications, haematological complications); binary (yes/no).

3. Severe PE: PE with blood pressure $\geq 110 / 160 \mathrm{~mm}$ $\mathrm{Hg}$, oliguria ( $<400 \mathrm{~mL} / 24$ hours), neurological symptoms, acute pulmonary oedema, persistent epigastric pain, hepatic dysfunction, analytical signs of haemolysis (lactate dehydrogenase $>700 \mathrm{U} / \mathrm{L}$ ), and/or thrombocytopenia $(<100000 / \mathrm{mL})$; binary (yes/no).

4. Preterm birth before 34 weeks of gestation; binary (yes/no).

5. Emergent caesarean section for loss of fetal well-being; binary (yes/no).

6. Birth weight; continuous $(\mathrm{g})$.

7. Neonatal acidosis (arterial $\mathrm{pH}<7.10+$ base excess $>12 \mathrm{mEq} / \mathrm{L}$ ); binary (yes/no) .

8. Perinatal mortality (from 22 weeks of gestation to 28 days post partum); binary (yes/no).

9. Days in the neonatal intensive care unit; continuous (days).

10. Neonatal morbidity (seizures, intraventricular haemorrhage grade III or IV, periventricular leukomalacia, hypoxic-ischaemic encephalopathy, abnormal electroencephalogram, necrotising entercholitis, acute renal failure (serum creatinine $>1.5 \mathrm{mg} / \mathrm{dL}$ ) or heart failure (requiring inotropic agents); binary (yes/no).

11. Biomarkers in maternal blood at the time of diagnosis and delivery: soluble fms-like tyrosine kinase 1 (sFlt) and placental growth factor (PlGF); continuous $(\mathrm{pg} / \mathrm{mL})$.

12. Biomarkers in umbilical cord blood: TNF- $\alpha$, IL-6, IFN- $\gamma$, FGF basic, VEGF and PlGF; continuous.

13. mRNAs in trophoblast: IL-6, IFN- $\gamma$, TNF- $\alpha$, VEGFA, VEGFB, FGF2, and RQVEGF Receptor1; continuous.

\section{PARTICIPANT TIMELINE}

Table 1 shows the participant timeline.

\section{SAMPLE SIZE}

A systematic review (PubMed and ISI Web of Knowledge) of clinical trials published in pregnant women suspected of placental insufficiency involving heparin treatment (both unfractionated and LMWH) only identified four studies with good or very good quality (Newcastle-Ottawa scale). The meta-analysis of these studies demonstrated very high heterogeneity under a fixed-effect model $\left(\mathrm{I}^{2} 90.4 \%\right)$ to estimate the difference in gestational age at birth between groups. Under a random effects model, the mean difference in gestational age at delivery was 1 week with an SD of 0.9. Under these assumptions, the sample needed to detect a clinically relevant difference (fixed at 1 week) at gestational age at delivery with a power of $95 \%$ and an alpha risk of $5 \%$ was 22 patients per arm (44 patients in total). To compensate for possible withdrawals, we will require 25 per arm (50 patients).

\section{ALLOCATION}

Using an online service (http://www.randomization. com), randomisation sequences will be generated in blocks of six subjects to ensure balanced distribution within study arms, stratified by participating site. Randomisation sequences will be also stratified for $<28$ and $\geq 28$ completed weeks and for umbilical artery Doppler with present or absent/reverse end diastolic flow. The allocation sequence will be internally sequestered by a CTU. At the time of the diagnosis and after enrolment, recruiting physicians will obtain the allocation group from a web-based system. Each treatment pack will only be identified by a randomisation code. The treatment allocation will only be revealed to the researchers after study completion or when clinically essential. The Pharmacy Department of Sant Joan de Déu Hospital will provide labelling for all packs and blister sheets, ensuring complete blinding to all participants and investigators including the principal investigator, participating research doctors, project managers and others involved in the trial. Matching placebo will be identical to the 


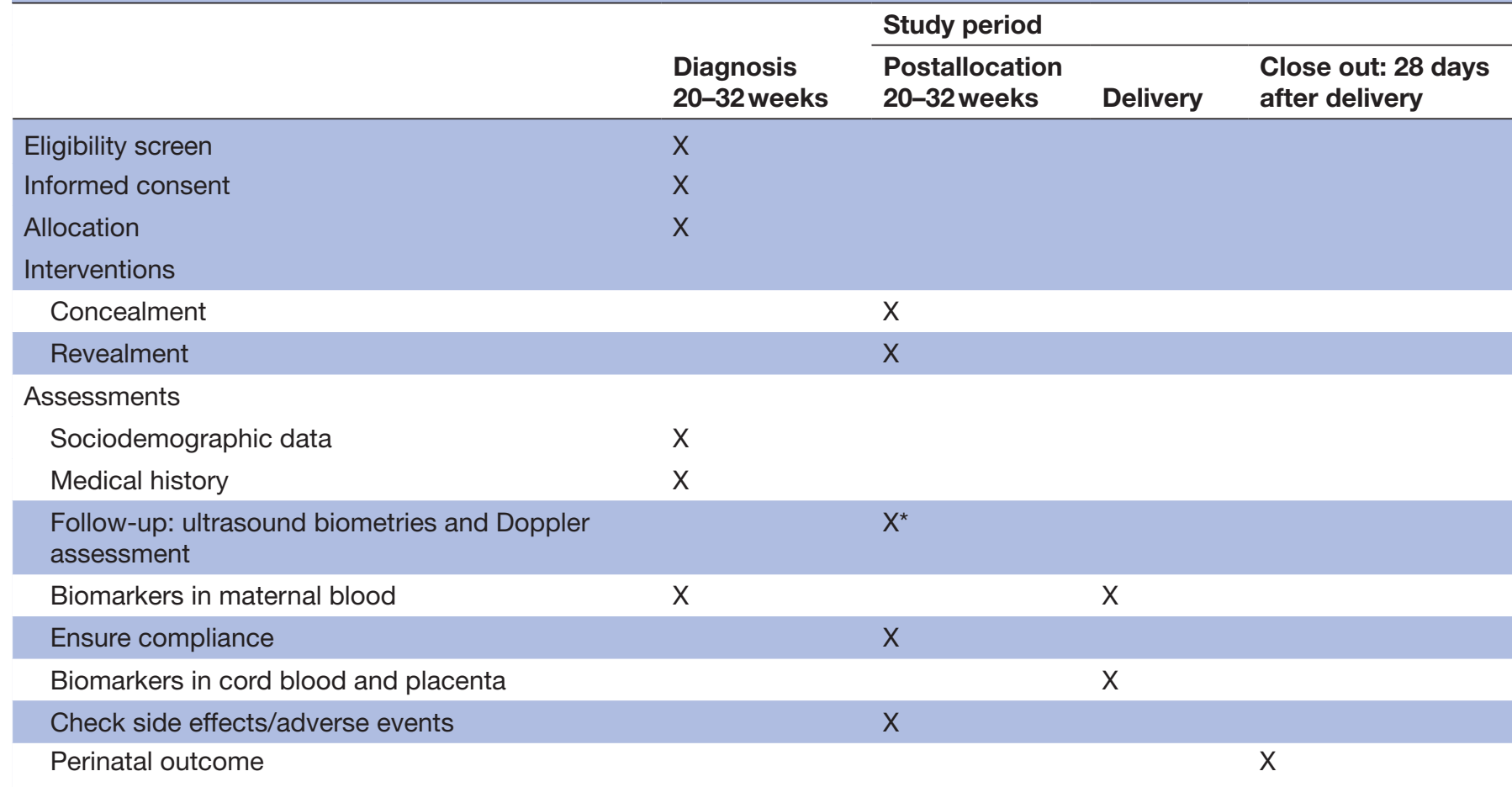

*According to local protocol (https://medicinafetalbarcelona.org/clinica/images/protocolos/patologia_fetal/cir-peg.pdf).

intervention (bemiparin) in such parameters as size, physical properties and appearance. The Pharmacy Department will keep the randomisation code list confidential.

\section{PARTICIPANT COMPLIANCE AND BLINDING PROCESS}

Compliance will be assessed by trial teams by counting remaining injections at each follow-up visit and inquiring about compliance weekly. Participants will be encouraged to report any concerns or side effects in a diary for review at each trial visit. Blinding success will be assessed before delivery by asking the managing doctor about suspicion of treatment arm (ie, due to the presence of bruising).

\section{DATA COLLECTION}

Patients will be recruited at the time of diagnosis of early onset IUGR, when fetal-placental Doppler study is routinely performed to obtain the mean PIs of the uterine arteries, umbilical artery, middle cerebral artery and ductus venosus. Maternal blood will be collected at the time of diagnosis to study angiogenic factors. The control variables defined in the previous section will also be collected in the first visit. Participant data from this first visit and subsequent visits for this study (anonymised) will be entered into an electronic case report form (e-CRF) hosted on a secure website by each site coordinator. Logic and range rules operate in the e-CRF to minimise imputation errors.

Maternal blood, cord blood and placenta will be collected at the time of delivery. Perinatal outcomes will be collected after delivery.

\section{Obtaining and processing of maternal blood samples}

We will collect $5 \mathrm{~mL}$ maternal blood in tubes without anticoagulant. They will be transported to the laboratory within 1 hour after extraction, maintaining each centre's safety guidelines for the transport of biological material. Blood tubes will be centrifuged at $1500 \mathrm{~g}$ for $15 \mathrm{~min}$. Supernatant (serum) will be aspirated, stored in cryophials, properly labelled and kept in a $-80^{\circ} \mathrm{C}$ freezer. Levels of PlGF and SFlt-1 (pg/mL) will be determined using an Elecsys automated platform by electrochemiluminescence (Cobas analyzers, Roche Diagnostics). The intra-assay and interassay coefficients of variation are $<4 \%$ and $2.4 \%-4.6 \%$, respectively.

\section{Collection and processing of cord blood samples}

After fetal extraction, umbilical cord venous blood will be collected in a $10 \mathrm{~mL}$ silicone tube without additives. Samples will be transported to the laboratory within 1 hour of extraction, maintaining our centre's safety guidelines for transport of biological material. Blood tubes will be centrifuged at $1500 \mathrm{~g}$ for $15 \mathrm{~min}$. Supernatant (serum) will be aspirated and stored in properly labelled cryophials in an $-80^{\circ} \mathrm{C}$ freezer until analysis in duplicate in the LABSCAN 100 Multiplex Analyzer (Luminex) (LUMINEX 100 IS software).

\section{Collection and processing of placental tissue samples}

After delivery, two placental tissue biopsies will be stored in later RNA in aliquots at $-80^{\circ} \mathrm{C}$ until the time of analysis. DNA will be extracted from fresh placental tissue with Flexigene (Qiagen). Placentas will then be fixed in buffered formalin. After gross examination, routine samples 
will be obtained from each specimen: one transverse section of cord, one rolled strip of membranes and three blocks from the placental parenchyma. Additional blocks will be taken from all macroscopic lesions. Placental findings will be reported as described in the Amsterdam Consensus. ${ }^{29}$

Maternal blood, cord blood and placental tissue samples will be analysed in the Centro de Investigación Biomédica de Aragón (CIBA), Aragon Institute for Health Research (IIS Aragón) Zaragoza, Spain.

\section{DATA MONITORING AND SAFETY}

An independent CTU will perform an offline data audit every 6 months checking for missing information and errors. The same committee will monitor safety issues. There may be unexpected adverse reactions associated with LMWH. All investigators have a thorough understanding of the anticipated adverse events and the appropriate reporting process. The investigators will notify the Independent CTU of adverse events, and they will report to the regulatory authority and ethics committee.

\section{PATIENT AND PUBLIC INVOLVEMENT}

Patients were not involved in any part of the design of the study.

\section{STATISTICAL ANALYSIS}

Analysis will be based on originally assigned groups (intention-to-treat). However, it is possible that there will still be some missing data at the end of the study, so sensitivity analyses will be carried out to confirm the robustness of the results.

The primary objectives will be analysed by linear regression model for gestational age and time from randomisation to live birth, in which the following covariates are considered: gestational age at inclusion (continuous) and PE (binary). Stillbirths will be considered as missing data will be addressed by multiple imputation methods. Stillbirth cases will be penalised in the analysis by imputing them 0 days of prolongation.

The number of patients included $(\sim 50)$ only allows adjustment by two variables to maintain power $>80 \%$.

Secondary objectives will be analysed by:

I. Univariate analysis:

A. Continuous variables: Student's t-test (or Mann-Whitney U tests for non-normal distribution, Shapiro-Wilk's test, $\mathrm{p}<0.05$ ).

B. Categorical variables: two Pearson's (or Fisher's exact test).

III. Multivariate analysis using linear regression (for continuous dependent variables) or logistic regression (for binary variables).

\section{ETHICS AND DISSEMINATION}

The study will be conducted in accordance with the principles of Good Clinical Practice. Results will be published in peer-reviewed journals and disseminated at international conferences.

Patients will be informed that their participation in the trial will be treated with the same confidentiality as their clinical documentation, but, if necessary, a member of the clinical research ethics committee of the centre, an inspector appointed by the health authorities or the clinical trial monitor may have access to it. In the data-collection notebook, the patient will only be identified by her study inclusion number.

The trial has been entered in the public registry www. clinicaltrial.gov according to Science Law 14/2011, and the results will be published in an open access journal.

\section{DISCUSSION}

The main limitation is the small sample size. However, the results will be very useful for designing larger, future trials, and it will provide mechanistic data on biomarkers in maternal blood, cord blood and placenta.

Although prolongation of pregnancy is a useful surrogate of perinatal health, the study would be largely underpowered if we set neonatal mortality/morbidity as a primary outcome.

\section{Author affiliations}

${ }^{1}$ BCNatal - Barcelona Center for Maternal-Fetal and Neonatal Medicine (Hospital Clínic and Hospital Sant Joan de Déu), University of Barcelona, Barcelona, Spain ${ }^{2}$ Institut de Recerca Sant Joan de Déu, Esplugues de Llobregat, Spain

${ }^{3}$ Maternal and Child Health and Development Network II (SAMID II), funded by Instituto de Salud Carlos III (ISCIII), Sub-Directorate General for Research Assessment and Promotion and the European Regional Development Fund (ERDF), Barcelona, Spain

${ }^{4}$ Fetal i+D Fetal Medicine Research Center, BCNatal - Barcelona Center for Maternal-Fetal and Neonatal Medicine (Hospital Clínic and Hospital Sant Joan de Déu), University of Barcelona, Barcelona, Spain

${ }^{5}$ Department of Pathology, Hospital Sant Joan de Déu, University of Barcelona, Barcelona, Spain

${ }^{6}$ Aragon Institute for Health Research (IIS Aragón), Obstetrics Department, Hospital Clínico Universitario Zaragoza, Zaragoza, Spain

${ }^{7}$ Institute for Health Sciences in Aragon (IACS), Aragon Institute for Health Research (IIS Aragón), Pluripotency in Embryonic Stem Cells group, Centro de Investigación Biomédica de Aragón (CIBA), ARAID Foundation, Zaragoza, Spain

${ }^{8}$ Department of Hemotherapy Hemostasis, Hospital Clinic de Barcelona, Barcelona, Spain

Contributors EM is the project monitor. NM, AP, DO, PI-B and JS are coordinators at their respective sites.EM is the general coordinator and principal investigator of the project. MC, CR, MDGR and MDT are consultants. FF is co-principal investigator of the study.

Funding This work is funded by the 'Plan Estatal de $1+D+I$ and Instituto de Salud Carlos III- Subdirección General de Evaluación y Fomento de la Investigación Sanitaria', project PI16/00151, and the European Regional Development Fund (FEDER).

Competing interests None declared.

Patient consent Obtained.

Ethics approval This protocol was approved by the Clinical Research Ethics Committee (CEIC) of Sant Joan de Déu Hospital on July 13th 2017. Subsequent approval by individual ethical committees and competent authority was granted.

Provenance and peer review Not commissioned; externally peer reviewed.

Open access This is an open access article distributed in accordance with the Creative Commons Attribution Non Commercial (CC BY-NC 4.0) license, which 
permits others to distribute, remix, adapt, build upon this work non-commercially, and license their derivative works on different terms, provided the original work is properly cited, appropriate credit is given, any changes made indicated, and the use is non-commercial. See: http://creativecommons.org/licenses/by-nc/4.0/.

\section{REFERENCES}

1. Figueras F, Gratacós E. Update on the diagnosis and classification of fetal growth restriction and proposal of a stage-based management protocol. Fetal Diagn Ther 2014;36:86-98.

2. Baschat $A A$, Cosmi E, Bilardo CM, et al. Predictors of neonatal outcome in early-onset placental dysfunction. Obstet Gynecol 2007;109:253-61.

3. Fisher SJ. The placental problem: linking abnormal cytotrophoblast differentiation to the maternal symptoms of preeclampsia. Reprod Biol Endocrinol 2004;2:53.

4. Hossain N, Paidas MJ. Adverse pregnancy outcome, the uteroplacental interface, and preventive strategies. Semin Perinatol 2007;31:208-12.

5. Hecher K, Bilardo CM, Stigter RH, et al. Monitoring of fetuses with intrauterine growth restriction: a longitudinal study. Ultrasound Obstet Gynecol 2001;18:564-70.

6. Cole TJ, Hey E, Richmond S. The PREM score: a graphical tool for predicting survival in very preterm births. Arch Dis Child Fetal Neonatal Ed 2010;95:F14-19.

7. Abheiden C, Van Hoorn ME, Hague WM, et al. Does low-molecularweight heparin influence fetal growth or uterine and umbilical arterial Doppler in women with a history of early-onset uteroplacental insufficiency and an inheritable thrombophilia? Secondary randomised controlled trial results. BJOG 2016;123.

8. Shomer E, Katzenell S, Zipori Y, et al. Microvesicles of pregnant women receiving low molecular weight heparin improve trophoblast function. Thromb Res 2016;137:141-7.

9. Shomer E, Katzenell S, Zipori Y, et al. Microvesicles of women with gestational hypertension and preeclampsia affect human trophoblast fate and endothelial function. Hypertension 2013;62:893-8.

10. Dunk $C$, Ahmed A. Expression of VEGF-C and activation of its receptors VEGFR-2 and VEGFR-3 in trophoblast. Histol Histopathol 2001;16:359-75.

11. Deng CL, Ling ST, Liu XQ, et al. Decreased expression of matrix metalloproteinase- 1 in the maternal umbilical serum, trophoblasts and decidua leads to preeclampsia. Exp Ther Med 2015;9:992-8.

12. Azizieh FY, Raghupathy RG. Tumor necrosis factor- $\alpha$ and pregnancy complications: a prospective study. Med Princ Pract 2015;24:165-70.

13. Ishihara $\mathrm{N}$, Matsuo $\mathrm{H}$, Murakoshi $\mathrm{H}$, et al. Increased apoptosis in the syncytiotrophoblast in human term placentas complicated by either preeclampsia or intrauterine growth retardation. Am J Obstet Gynecol 2002;186:158-66.

14. Dodd JM, McLeod A, Windrim RC, et al. Antithrombotic therapy for improving maternal or infant health outcomes in women considered at risk of placental dysfunction. Cochrane Database Syst Rev 2013;24:CD006780.

15. Roberge S, Demers S, Nicolaides KH, et al. Prevention of preeclampsia by low-molecular-weight heparin in addition to aspirin: a meta-analysis. Ultrasound Obstet Gynecol 2016;47:548-53.

16. Rodger MA, Gris JC, de Vries JIP, et al. Low-molecular-weight heparin and recurrent placenta-mediated pregnancy complications: a meta-analysis of individual patient data from randomised controlled trials. Lancet 2016;388:2629-41.

17. Kingdom JC, Walker M, Proctor LK, Shah M, et al. Unfractionated heparin for second trimester placental insufficiency: a pilot randomized trial. J Thromb Haemost 2011;9:1483-92.

18. D'Souza R, Keating S, Walker M, et al. Unfractionated heparin and placental pathology in high-risk pregnancies: secondary analysis of a pilot randomized controlled trial. Placenta 2014;35:816-23.

19. Yu YH, Shen LY, Zhong M, et al. [Effect of heparin on fetal growth restriction]. Zhonghua Fu Chan Ke Za Zhi 2004;39:793-6.

20. Yu YH, Shen LY, Zou H, et al. Heparin for patients with growth restricted fetus: a prospective randomized controlled trial. $J$ Matern Fetal Neonatal Med 2010;23:980-7.

21. Gordijn SJ, Beune IM, Thilaganathan B, et al. Consensus definition of fetal growth restriction: a Delphi procedure. Ultrasound Obstet Gynecol 2016;48:333-9.

22. Anon. Editrial/Pregnancy Hypertension. Int J Women Cardio Healt 2014;4:97-104

23. Figueras F, Meler E, Iraola A, et al. Customized birthweight standards for a Spanish population. Eur J Obstet Gynecol Reprod Biol 2008;136:20-4.

24. Gómez O, Figueras F, Fernández S, et al. Reference ranges for uterine artery mean pulsatility index at 11-41 weeks of gestation. Ultrasound Obstet Gynecol 2008;32:128-32.

25. Arduini D, Rizzo G. Normal values of Pulsatility Index from fetal vessels: a cross-sectional study on 1556 healthy fetuses. J Perinat Med 1990;18:165-72.

26. Baschat AA, Gembruch U. The cerebroplacental Doppler ratio revisited. Ultrasound Obstet Gynecol 2003;21:124-7.

27. Hecher K, Campbell S, Snijders R, et al. Reference ranges for fetal venous and atrioventricular blood flow parameters. Ultrasound Obstet Gynecol 1994;4:381-90.

28. Robinson HP, Fleming JE. A critical evaluation of sonar crown-rump length measurements. Br J Obstet Gynaecol 1975;82:702-10.

29. Khong TY, Mooney EE, Ariel I, et al. Sampling and definitions of placental lesions: Amsterdam placental workshop group consensus statement. Arch Pathol Lab Med 2016;140:698-713. 